\title{
The Fraction of Runaway OB Stars in the SMC Field
}

\author{
J. B. Lamb ${ }^{1}$ and M. S. Oey ${ }^{1}$ \\ ${ }^{1}$ Department of Astronomy, University of Michigan, Ann Arbor, MI 48109-1042, USA \\ email: joellamb@umich.edu
}

\begin{abstract}
The fraction of field OB stars that originate from clusters can help probe the dynamical evolution of clusters. Field stars represent a significant fraction (20-30\%) of the OB population in galaxies, and estimates for the fraction of field OB stars that are runaways range from the classical value of $<10 \%$ (Blaauw 1961) to contemporary results suggesting $>90 \%$ (de Wit et al. 2005). We obtained Magellan IMACS observations on the kinematics of field OB stars in the SMC to examine the line-of-sight velocities of this population. Using these observations, we will estimate the fraction of runaways to serve as a probe of cluster evolution.
\end{abstract}

Keywords. binaries: general, stars: early-type, stars: kinematics, stars: statistics, open clusters and associations: general, galaxies individual (Magellanic Clouds), galaxies: stellar content

Cluster evolution is heavily influenced by the massive stars formed in the cluster. Ionizing radiation and hot star winds can unbind clusters on the order of dynamical timescales $(\leqslant 1 \mathrm{Myr})$ by dispersing gas from the cluster. In some cases, a mass loss as little as $10 \%$ can dissociate a cluster (Hills 1980). These feedback effects also affect the rate and efficiency of star formation on timescales of $\sim 1$ Myr (Dale et al. 2005). In addition, on time scales $>3 \mathrm{Myr}$, supernova feedback can further affect the binding and star formation of the cluster (Dray et al. 2005). The fraction of OB stars that leave clusters as runaways is important, not only because of the stellar dynamical effects, but also because these runaways deprive clusters of their feedback effects.

Runaways are created primarily through two possible mechanisms. The Dynamical Ejection Scenario (DES; Poveda, Ruiz \& Allen 1967) involves the interactions of primarily binary-binary pairs in the cores of star clusters (Leonard \& Duncan 1990). These interactions typically result in the less massive star of one of the binaries being ejected from the system. The other runaway-generating mechanism is the Binary Supernova Scenario (BSS; Blaauw 1961), in which the supernova of one of the stars in an early-type binary leaves the other star as a runaway, sometimes with the compact remnant still bound. As in DES, the less massive star of the binary pair ends up as the dominant runaway. Due to binary evolution, BSS-generated runaways should have large rotational velocities (Hoogerwerf, de Bruijne \& de Zeeuw 2001). Portegies Zwart (2000) finds that the rotational velocity is expected to be higher with increasing mass.

For DES, the OB stars are ejected at a very young age, $<1$ Myr. This deprives the cluster of nearly all massive star feedback from the runaway star. However, for BSS, the supernova requirement necessitates that the more massive star in the binary pair must complete its life cycle for ejection to occur. This requirement leads to "older" stars $(3-40 \mathrm{Myr})$ being ejected for BSS. Thus, for BSS, much of the massive star feedback from the runaways is still imparted to the cluster. Therefore, not only the fraction of runaways, but also the method of runaway ejection, affects the evolution of clusters. However, neither of these mechanisms is well understood. Few papers provide 
comparisons of the likelihood of one ejection scenario versus the other, but one estimate is that BSS dominates by a 2:1 ratio (Hoogerwerf, de Bruijne \& de Zeeuw 2001).

Field stars represent $20-30 \%$ of the OB population in galaxies (Oey, King, \& Parker 2004). The percentage of these field stars that are thought to be runaways varies considerably, with estimates from 10\% (Blaauw 1961) to $90 \%$ (de Wit et al. 2005). Combining these estimates yields the result that anywhere from $2 \%$ to $27 \%$ of OB stars created in clusters become runaways. However, studies of specific clusters show evidence of ejection rates as high as $75 \%$ (Pflamm-Altenburg \& Kroupa 2006).

All previous empirical studies of runaway stars focus on samples in the local Galactic neighborhood where gas and dust cause line-of-sight confusion and uncertainties in distances (e.g., Gies 1987). Observing the SMC circumvents these issues since it lies outside the Galactic plane, allowing us to obtain a virtually spatially complete sample of the SMC bar. We choose target stars from a sample of candidate field OB stars with estimated spectral types B0 V, B0.5 I and earlier (Oey, King, \& Parker 2004; and in these Proceedings). Multi-slit spectra of $\sim 150$ stars within the SMC bar were taken with the Magellan IMACS multi-object spectrograph.

Preliminary data for two fields $\left(15^{\prime} \times 15^{\prime}\right.$ each $)$ containing a total of sixteen targets were found to have a radial velocity range of $102 \mathrm{~km} / \mathrm{s}$ with a velocity resolution in the 15-20 km/s range. The SMC systemic velocity of $158 \mathrm{~km} / \mathrm{s}$ gives the sample a velocity range of $-49 \mathrm{~km} / \mathrm{s}$ to $+54 \mathrm{~km} / \mathrm{s} \pm 20 \mathrm{~km} / \mathrm{s}$. Comparing with known velocity dispersions such as typical OB associations (10 km/s), SMC HI (10-40 km/s; Stanimirovic, StaveleySmith \& Jones 2004), and runaway populations (30 km/s; Stone 1991) gives an initial impression that runaway stars may be present in this sample. Five of the sixteen stars have velocities exceeding the $30 \mathrm{~km} / \mathrm{s}$ threshold velocity for runaway status.

With our complete dataset, we will estimate the fraction of runaways among field OB stars. We will test the applicability of the two ejection methods: DES ejection predicts the lowest mass stars to have the highest runaway velocities (Leonard \& Duncan 1990), while BSS predicts the opposite (Stone 1991). Finally, we will measure the rotational velocities to test the predicted correlation of rotational speed with mass, as expected for BSS (Portegies Zwart 2000). We will obtain an estimate for the fraction of stars produced by each ejection method, and examine the consequences for cluster evolution.

\section{References}

Blaauw, A. 1961, Bull. Astron. Inst. Netherlands 15, 265

Dale, J. E., Bonnell, I. A., Clarke, C. J., \& Bate, M. R. 2005, MNRAS 358, 291

de Wit, W. J., Testi, L., Palla, F., \& Zinnecker, H. 2005, A\&A 437, 247

Dray, L. M., Dale, J. E., Beer, M. E., Napiwotzki, R., \& King, A. R. 2005, MNRAS 364, 59

Gies, D. R. 1987, ApJS 64, 545

Hills, J. G. 1980, ApJ 225, 986

Hoogerwerf, R., de Bruijne, J. H. J., \& de Zeeuw, P. T. 2001, A\&SA 365, 49

Leonard, P. J. T. \& Duncan, M. J. 1990, ApJ 99, 608

Oey, M. S., King, N. L., \& Parker, J. 2004, AJ 127, 1632

Pflamm-Altenburg, J. \& Kroupa, P. 2006, MNRAS 373, 296

Portegies Zwart, S. F. 2000, ApJ 544, 437

Poveda, A., Ruiz, J., \& Allen, C. 1967 Bol. Obs. Tonantzinla Tacubaya 28, 68

Stanimirovic, S., Staveley-Smith, L., \& Jones, P. A. 2004, ApJ 604, 176

Stone, R. C. 1991, AJ 102, 333 